\title{
Complete Response with Re-Irradiation in Recurrent Pediatric Ependymoma - A Case Report
}

\section{Anil Kumar Anand, Charu Garg, Gaurav Kumar ${ }^{*}$ and Anil Kumar Bansal}

Department of Radiation Oncology, Max Super specialty hospital, Max Cancer Centre, Saket, New Delhi, India

"Corresponding author: Gaurav Kumar, Department of Radiation Oncology, Max Super specialty hospital, Max Cancer Centre, Saket, New Delhi, India, Tel: + 91-11-26515050; E-mail: dr_gauravkumar@yahoo.com

Received date: Dec 05, 2014, Accepted date: Jan 08, 2015, Publication date: Jan 15, 2015

Copyright: ( 2015 Anand AK, et al. This is an open-access article distributed under the terms of the Creative Commons Attribution License, which permits unrestricted use, distribution, and reproduction in any medium, provided the original author and source are credited.

\begin{abstract}
Ependymoma accounts for $5 \%$ to $10 \%$ of all brain in the paediatric age group. The mainstay of the treatment is surgery followed by radiotherapy. The primary site remains the most common site $(85 \%)$ for the recurrences followed by the metastatic disease $(25 \%)$. Here we present a case report of a 5 year old male child who presented as an operated case of ependymoma and was treated with localized radiation therapy. After a disease free interval of one year the child relapsed in the brain and spine. He was re operated for the brain lesion and then was taken up for cranio spinal irradiation. This was followed by systemic chemotherapy. The follow up scans should complete resolution of the disease in the brain and the spine. Hence, longer follow up is needed to appreciate the long term effectiveness and toxicity of this treatment protocol.
\end{abstract}

Keywords: Re-irradiation; Recurrent pediatric ependymoma

\section{Introduction}

Ependymoma accounts for $5 \%$ to $10 \%$ of all brain tumors in paediatric age group, with predilection for children younger than 5 years [1]. The mainstay of treatment is surgery followed by local radiotherapy, provided there are no lepto-meningeal seedings. Improvements in surgical and radiation therapy techniques have resulted in better survival with 5 year event free survival rate of more than $70 \%$ [2]. However, patients with recurrent ependymoma have poor survival ( $25 \%$ at 5 years). The primary site remains the most common site $(85 \%)$ of recurrence followed by metastatic disease $(25 \%)$ [3]. Site of relapse has significant relation to the outcome, with locally recurring tumors having better outcome than those with metastatic relapse (median 30 months versus 13 months, $p=0.02$ ) [3]. In cases of relapse the treatment options available are: chemotherapy, resurgery and fractionated reirrdiation. Here we present a case of ependymoma with recurrence in cranio-spinal axis, after a disease free interval of one year and was treated with re-irradiation.

\section{Case Report}

A 5 year old male child presented with history of vomiting, dimunition in vision and mild right eye squint in April, 2010. He was evaluated with MRI which showed a space occupying lesion in left posterior parieto-occipital region measuring $8.4 \times 5.5 \times 7 \mathrm{~cm}$, with intra-lesional calcification. He underwent gross total removal of tumor using real time neuronavigation. The histopathology report was Anaplastic Ependymoma, WHO grade III.

Post operatively T1W, T2W and FLAIR MRI brain was done which showed complete removal of the tumor. MRI spine screening and CSF analysis revealed no abnormality. He received $60.1 \mathrm{~Gy}$ in 30 fractions to the tumor bed and $53.1 \mathrm{~Gy}$ in 30 fractions to the planning target volume by intensity modulated radiotherapy under image guidance from April to June, 2010 (Figure 1). He tolerated the treatment well.

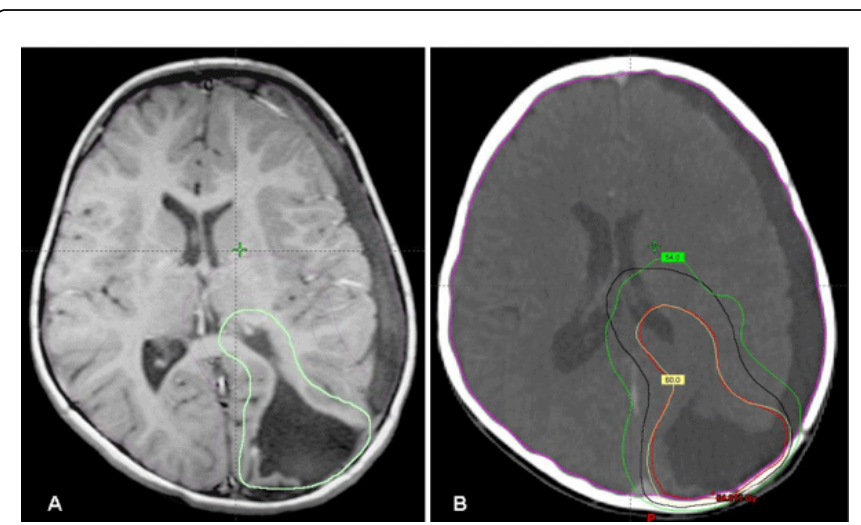

Figure 1: T1W contrast axial MRI scan showing post operative cavity and the clinical target volume marked in green (A); Planning CT scan showing the isodose curves (B).

The child was kept on regular follow up with 3 monthly clinical and radiological examinations. However, after a disease free interval of 12 months he presented with unsteady gait in June 2011.

The T1W contrast axial MRI brain revealed large mildly enhancing extra - axial mass lesion in left cerebello pontine angle causing mass effect on brain stem and cerebellum with partial effacement of fourth ventricle. However there was no evidence of recurrent lesion at the site of original tumor. Screening MRI whole spine was done on 1.5Tesla unit and the images were acquired in axial, coronal and sagittal planes on T1W, T1C, T2W \& FLAIR sequences which revealed multiple enhancing nodular lesions in spinal cord extending from C2 to D1 levels and along the cauda equina nerve roots (Figure 2). 

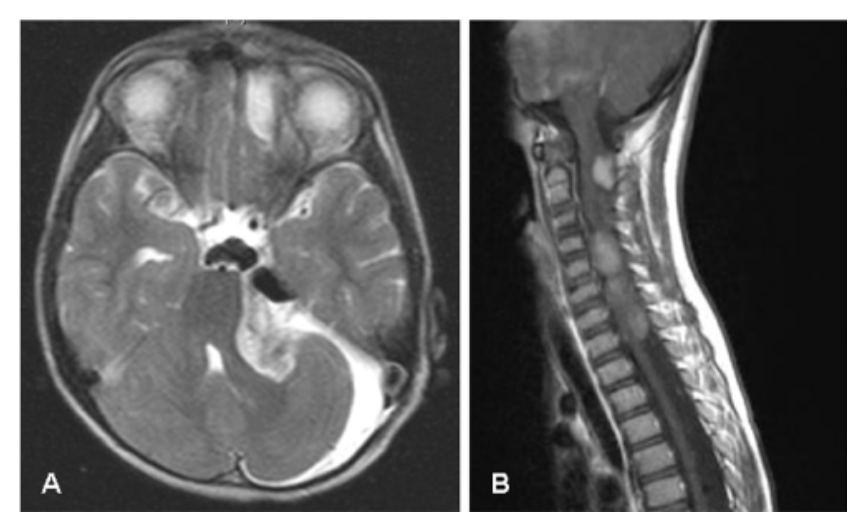

Figure 2: T2W axial MRI brain showing recurrence at left cerebellopontine angle (A); T1W contrast sagittal MRI spine showing metastatic seedings from C2 to D1 levels (B).

The case was discussed in neuro-oncology clinic. A decision of resurgery followed by radiotherapy to cranio-spinal axis and chemotherapy was taken. He underwent gross total excision of the brain tumour. The post operative histopathology was again consistent with Anaplastic Ependymoma, WHO grade III.

The child received craniospinal irradiation with boost to gross disease. He received 39.1Gy in 23 fractions to whole spine, 30.6Gy in 18 fractions to whole brain followed by boost of $3.4 \mathrm{~Gy}$ in 2 fractions to gross spinal lesion and $19.8 \mathrm{~Gy}$ in 11 fractions to brain lesion in September 2011 (Figure 3). Dose constraints were dictated by tolerance of various structures compromised by previous radiotherapy. He tolerated the treatment well.

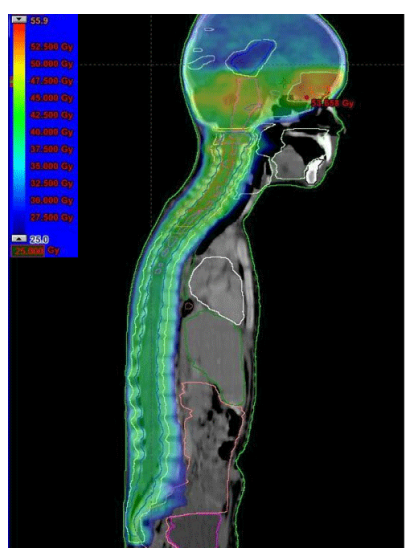

Figure 3: Radiotherapy planning CT scan showing cranio spinal radiation isodose distribution.

After four weeks, adjuvant chemotherapy with Ifosphosphamide, Etoposide, Carboplatin and Mesna was started. Till May, 2012 he received 8 cycles of chemotherapy. The child is clinically and radiologically disease free except right sided facial paraesis which had developed after second surgery.

MRI scans done in November, 2011 and February, 2012 showed complete resolution of brain and spinal lesions (Figure 4).

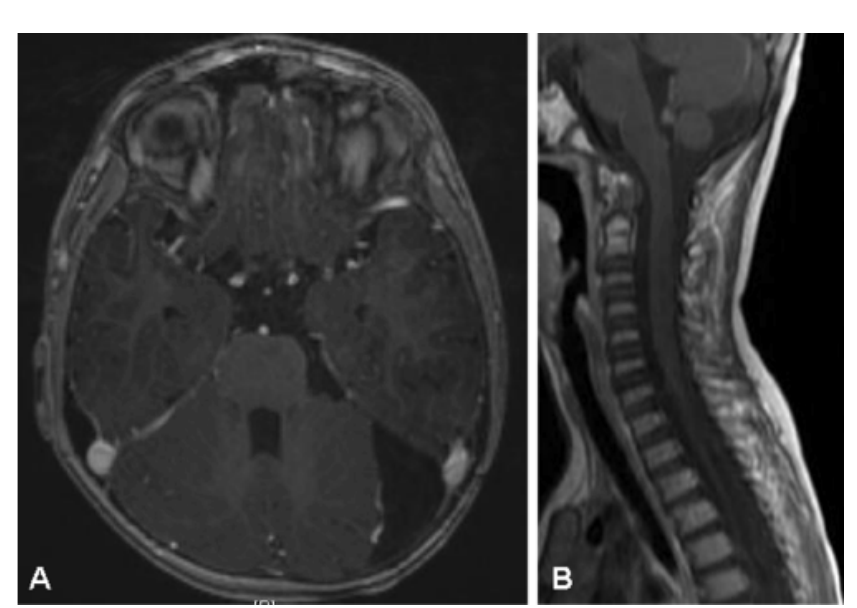

Figure 4: Follow up T1W contrast axial MRI brain (A) and T1W contrast sagittal spine (B) showing complete response.

\section{Discussion}

Historically the commonest pattern of failure in treated ependymoma with surgery and radiotherapy was local. With improvements in surgical techniques and image guided high dose irradiation, the failures have become mixed that is local and/or metastatic [2].

In a recently published retrospective study of 113 patients, $41.2 \%$ patients relapsed [4]. Sixty one percent of the relapsed patients were treated with surgery and /or chemotherapy while $38.8 \%$ were reirradiated to a full course of radiotherapy (focal and /or craniospinal). The 3 year overall survival was $7 \% \pm 6 \%$ versus $81 \% \pm$ $12 \%$ for nonreirradiated and reirradiated patients respectively $(\mathrm{p}<0.0001)$. The same study also reported that in ependymomas the time to second progression after reirradiation is longer than time to first progression. This striking clinicobiological observation suggests a mechanism of hypersenstization or a cumulative toxicity of ependymoma cells to the second dose of radiation. The 3 year progression free survival was $61 \% \pm 15 \%$ after reirradiation versus $25 \%$ $\pm 11 \%$ at first relapse $(\mathrm{p}=0.03)$ [4].

It is seen that children with infratentorial lesions and younger age group do worse.

Another reported retrospective study of recurrent ependymoma included 38 paediatric patients with initial localized ependymoma who received limited field radiotherapy [5]. All of them received a second course of radiotherapy after local $(n=21)$, metastatic $(n=13)$, or combined $(\mathrm{n}=4)$ failures. Thirteen patients with local failures underwent focal fractionated radiotherapy to median combined dose of $111.6 \mathrm{~Gy}$. Three patients progressed at a median of 6 months while 10 patients had no evidence of disease (median follow up of 30 months). Nineteen patients received cranio-spinal irradiation, of whom 9 patients had disease free interval of 22 months and 1 patient remained disease free for more than 20 years. Rest 6 patients were treated with radiosurgery to median dose of 18 Gy. Four patients experienced local and or distant failures and died, all of them had radiological or pathological evidence of necrosis. One patient died at 40 months and one patient remained disease free at 10 years. 
With this kind of encouraging observation with re-irradiation, we treated the child with re-irradiation to Craniospinal axis. He achieved complete reeponse in both cranial and spinal relapse of the tumor. It corroborates the observation of Bouffet et al and Merchant et al, where high response rates have been reported with re-irradiation. Fractionated stereotactic radiotherapy is well tolerated and is highly effective in the management of ependymoma in primary as well as in reirradiation cases [6].

In conclusion, the recurrent cases of ependymoma should undergo resection of local and metastatic disease to the maximum possible extent. This should be followed by reirradiation with fractionated stereotactic radiotherapy with curative intent respecting the tolerance of critical organs. However longer follow up is needed to appreciate the long term effectiveness and toxicity of this treatment protocol.

\section{References}

1. Freeman CR, Farmer JP, Taylor RE (2008) Central Nervous system tumors in children. Principles and Practice of Radiation Oncology. Philadelphia: Walter Kluwer, Lippincott Williams\& Wilkins: 1822-1849.
2. Merchant TE, Mulhern RK, Krasin MJ, Kun LE, Williams T, et al. (2004) Preliminary results from a phase II trial of conformal radiation therapy and evaluation of radiation-related CNS effects for paediatric patients with localized ependymoma. J Clin Oncol 22: 3156-3162.

3. Messahel B, Ashley S, Saran F, Ellison D, Ironside J, et al. (2009) Relapsed intracranial ependymoma in children in the U.K: patterns of relapse, survival and therapeutic outcome. Eur J Cancer 45: 1815-1823.

4. Bouffet E, Hawkins CE, Ballourah W, Taylor MD, Bartels UK, et al. (2012) Survival benefit for paediatric patients with recurrent ependymoma treated with reirradiation. Int J Radiat Oncol Biol Phys 83: 1541-1548.

5. Merchant TE, Boop FA, Kun LE, Sanford RA (2008) A retrospective study of surgery and reirradiation for recurrent ependymoma. Int $\mathrm{J}$ Radiat Oncol Biol Phys. 71: 87-97.

6. Combs SE, Thilmann C, Debus J, Schulz-Ertner D (2006) Local radiotherapeutic management of ependymomas with fractionated stereotactic radiotherapy. BMC Cancer 6: 222. 